\title{
Immunotherapy and target therapy in non-hodgkin lymphoma
}

\author{
Nepton Sheik Khoni ${ }^{*}$, Alaa Ahmed ${ }^{2}$, Ghazaleh Shoja E Razavi ${ }^{1}$ and Abdullateef Al-Hadeethi ${ }^{3}$ \\ ${ }^{1}$ Global Allied Pharmaceutical, Center for Excellence in Research and Development, 160 Vista Oak Dr. Longwood, FL 32779, USA \\ ${ }^{2}$ Immunopathology and Laboratory Medicine, Chief Scientific Officer and medical Research Center, Jazan University, Jazan, KSA \\ ${ }^{3}$ Global Allied Pharmaceuticals, Gardens St, Amman, 11195, Jordan
}

\begin{abstract}
Non-Hodgkins's lymphomas (NHL) are a heterogenous group of B and T cell malignancies primarily involving lymphatic system. NHL is fifth leading cause of cancer mortality. Due to evolving science and better understanding of pathophysiology of the disease leading to the subsequent development of newer targeted agents, the potentially curable variants of NHL are increasing. In this paper, we will discuss the current trends in utilizing immunotherapy to fight NHL along with the different molecules involved in the proliferation of the disease.
\end{abstract}

\begin{abstract}
Abbreviations: NHL: Non-Hodgkins lymphoma; MABs: Monoclonal antibodies; FDA: Food and Drug Administration; Mtor: Mammalian Target of Rapamycin Immunotherapy; IFN: Interferon; IL: Interleukin; NK: natural killer; HLA: Human leukocyte antigen; ADC: antibody drug conjugate; PML: Progressive Multifocal Leukoencephalopathy
\end{abstract}

\section{Introduction/Epidemiology}

According to the SEER statistics, in the year 2014, 70,800 new cases (32,530 in women and 38,270 in men) of non-Hodgkin's lymphoma (NHL) were estimated. Additionally, in the same year, 18,990 deaths ( 8,520 women and 10,470 men) due to NHL in the United States were also estimated. The annual rate of new cases was 19.7 per 100,000 men and women and the deaths rate was 6.3 per 100,000 men and women. These are age-adjusted and also based on the cases and deaths from the year 2007-2011. It was estimated that 530,919 people were alive with this disease in the US in the year 2011. The five year survival rate of patients is $69.3 \%$ after the diagnosis of NHL and it also represents $4.3 \%$ of all the cancers in the United States [1]. The average risk of development of NHL during the lifetime is about 1 in 50 persons. The NHL can arise in both children and adults of any age. However, around half of the patients are older than 66 years [2]. There is a 1.3:1 predominance of male over female [3]. The availability of new technology in diagnosis helps in the early detection of NHL.

NHL is a heterogeneous group of disorders, which includes malignant monoclonal proliferation of lymphoid cells in different lymphoreticular sites, including GI tract, spleen, lymph nodes, liver and bone marrow. The most common signs and symptoms of NHL are chest pain or nonproductive dry cough, enlarged lymph nodes, weight loss, fever, low red blood cell counts (anemia), night sweats, swollen abdomen (belly), fatigue (extreme tiredness), and feeling full after only a small amount of food.

Factors such as gender, age, race, ethnicity, geography, radiation exposure, exposure to certain chemicals such as benzene, HIV, hepatitis
C virus, Epstein-Barr virus and human T-cell leukemia-lymphoma virus have been proposed as a predisposing factor for certain types of lymphomas [2]. There are also some genetic predispositions and chronic inflammatory situations that are also responsible for the NHL. Other risk factors include skin cancer and ultraviolet radiation exposure, autoimmune and chronic inflammatory disorders, tobacco smoking, occupational exposures, allergy, anthropometric measures, blood transfusion, alcohol and dietary factors, hair dyes, medication, and other environmental factors [4,5]. The chromosomal abnormality in NHL comprises of chromosomal translocation, chromosomal deletion and various mutations.

\section{Immunotherapeutic in NHL}

\section{Monoclonal antibody (MAB) immunotherapy}

Rituximab [6]: A recombinant chimeric murine/human antibody directed against the CD20 antigen, a hydrophobic transmembrane protein located on normal pre- $\mathrm{B}$ and mature $\mathrm{B}$ lymphocytes. Rituximab is a CD20-directed cytolytic antibody for the treatment of patients with NHL. It is specifically approved for the first line treatment of patients with diffuse large B-cells, CD20 positive, NHL in combination with cyclophosphamide, doxorubicin, vincristine and prednisone (CHOP) or other anthracycline-based chemotherapy regimens. It is also approved for the first-line treatment of patients with low grade or follicular B-cell, CD20-positive NHL. It is approved either in combination with CVP chemotherapy (cyclophosphamide, vincristine and prednisone); or following CVP chemotherapy as a maintenance

Correspondence to: Nepton Sheik Khoni, Global Allied Pharmaceutical, Center for Excellence in Research and Development, 160 Vista Oak Dr. Longwood, FL 32779, USA, Tel: 1-321-945-4283; E-mail: timothy.allen@gapsos.com

Key words: non-hodgkins lymphoma, immunotherapy, immunosuppression, kinase inhibitors, cytokine, multiclonal antibodies, interleukins, proteasome inhibitors

Received: June 10, 2016; Accepted: June 22, 2016; Published: June 25, 2016 
therapy for Follicular CD-20 Positive B-cell NHL. The half-life of Rituximab is 32 days.

The most common adverse effects are fever, infection, infusion reactions, asthenia, and lymphopenia. Rituximab can cause severe hypersensitivity reactions as well as fatal, infusion reactions. Tumor Lysis Syndrome can take place after 12-24 hours. Severe Mucocutaneous Reactions including Stevens-Johnson syndrome, paraneoplastic pemphigus, and vesiculobullous dermatitis are notedRituximab is is contraindicated with Progressive Multifocal Leukoencephalopathy (PMLE) and shoulddiscontinued in Hepatitis B virus (HBV) reactivation, infections such as bacterial, fungal, and fatal, new or reactivated viral infections.

Ofatumumab [7]: A fully human, high-affinity IgG1 monoclonal antibody directed against the B cell CD20 cell surface antigen with potential anti-neoplastic activity. Ofatumumab binds specifically to CD20 on the surfaces of B cells, triggering complement-dependent cell lysis (CDCL) and antibody-dependent cell-mediated cytotoxicity (ADCC) of B cells over expressing CD20. The CD20 antigen, found on over $90 \%$ of B cells, B cell lymphomas and other B cells of lymphoid tumors of B cell origin, is a non-glycosylated cell surface phosphoprotein that acts as a calcium ion channel; it is exclusively expressed on B cells during most stages of B cell development. The half-life is 15.6 days.

Ofatumumab hhas been approved for chronic lymphocytic leukemia patients both as a first line treatment in combination with chlorambucil and in previously treated patients. The most common adverse effects are neutropenia, infusion reactions, cough, anemia, dyspnea, diarrhea, pneumonia, fatigue, nausea, and upper respiratory tract infections. HBV reactivation has occurred in patients treated with Ofatumumab. Ofatumumab should be discontinued in patients with Progressive Multifocal Leukoencephalopathy (PML) as it may lead to death. Severe cytopenias like thrombocytopenia, anemia and neutropenia can occur with Ofatumumab in patients who received chlorambucil in combination.

Obinutuzumab [8]: A third generation type II anti-CD20 antibody, selectively binds to the extracellular domain of the human CD20 antigen on malignant human B cells. The FC region carbohydrates of the antibody, enriched in bisected non-fucosylated glycosylation variants, contribute to its higher binding affinity for human FC gamma RIII receptors compared to non-glycoengineered antibodies, resulting in enhanced antibody-dependent cellular cytotoxicity (ADCC) and caspase-independent apoptosis. In addition, modification of elbow hinge sequences within the antibody, variable framework regions may account for the strong apoptosis-inducing activity of R7159 upon binding to CD20 on target cells. The half-life is 28.4 days.

Obinutuzumab has been approved ndicated for previously untreated chronic lymphocytic leukemia in combination with chlorambucil. It is also approved as a second line treatment on combination with bendamustin in patients with follicular lymphoma previously treated with rituximab anti CD20 monoclonal antibody. It is an orphan designated drug for marginal zone splenic B cell lymphoma as well.

The most common adverse effects are anemia, thrombocytopenia, musculoskeletal disorder, neutropenia, cough, infusion reactions, and pyrexia. HBV re-activation has occurred in patients treated with Obinutuzumab. Obinutuzumab should be discontinued in patients with Progressive Multifocal Leukoencephalopathy (PML) as it may lead to death. Tumor Lysis Syndrome can take place after 12-24 hours of administration of Obinutuzumab.

Ibritumomab tiuxetan [9]: Ibritumomab is a murine IgG1 kappa monoclonal antibody directed against the CD20 antigen, which is found on the surface of normal and malignant B lymphocytes. When bound to indium In 111 or yttrium Y 90, ibritumomab tiuxetan, targeting the CD20 antigen on B cell surfaces, specifically delivers a potentially cytotoxic dose of radiation to B lymphocytes.

Ibritumomab is indicated for the treatment of patients with relapsed or refractory low-grade, follicular, or transformed B-cell NHL, including patients with Rituximab refractory follicular NHL. The most common adverse effects are neutropenia, cough, anemia, dyspnea, diarrhea, pneumonia, nausea, anorexia, ecchymosis, anxiety and arthralgia. Ibritumomab should not be given in patients who have less than $25 \%$ of lymphoma marrow or impaired bone marrow reserve. Ibritumomab can cause fetal harm and should be avoided in pregnant women.

Tositumomab iodine I 131[10]: A murine IgG2a lambda monoclonal antibody, both unradiolabeled and radiolabeled with iodine I 131, directed against the human B cell-specific surface antigen CD20. Tositumomab binds to CD20, resulting in complementdependent cytotoxicity (CDC), ADCC and apoptosis in B cells expressing CD20; in addition to the antibody-mediated cytotoxicity, iodine I 131 tositumomab delivers cytotoxic ionizing radiation specifically to CD20-expressing B cells. In a two-step therapeutic regimen, a predose of un-radiolabeled tositumomab is administered first, followed by the administration of a dosimetric dose of iodine I 131 tositumomab; 7-14 days later, a therapeutic dose of iodine I 131 tositumomab is administered. The predose of un-radiolabeled tositumomab binds to non-tumor B cells, increasing the terminal halflife of radiolabeled antibody while protecting non-tumor B cells from radiolabeled antibody-mediated radio cytotoxicity.

Tositumomab and Iodine I 131 Tositumomab is indicated for the treatment of patients with CD20 positive, follicular, NHL, with and without transformation, whose disease is refractory to Rituximab and has relapsed following chemotherapy. This medication should be intended as a single course of treatment. The safety of multiple courses of the Tositumomab Iodine therapeutic regimen, or combination of this regimen with other forms of irradiation or chemotherapy, has not been evaluated. It is contraindicated in patients with hypersensitivity to murine proteins or any other component of the Tositumumab Iodine.

The most common adverse effects are anaemia, thrombocytopenia, neutropenia dehydration, pleural effusion, hypothyroidism, immunogenicity, secondary leukemia, and myelodysplastic syndrome Patients experienced thrombocytopenia and neutropenia when Tositumomab is administered. The Tositumomab should not be given to patients with $>25 \%$ lymphoma marrow association or impaired bone marrow replacement. Tositumomab should not be administered in pregnant women because it can cause fetal harm. GlaxoSmithKline announced the discontinuation of the manufacture and sale of the BEXXAR $^{\oplus}$ therapeutic regimen (tositumomab and iodine I 131 tositumomab) on February 20, 2014.

Brentuximab vedotin [11]: An antibody-drug conjugate (ADC) directed against the tumor necrosis factor (TNF) receptor CD30 with potential anti-neoplastic activity. Brentuximab vedotin is generated by conjugating the humanized anti-CD30 monoclonal antibody SGN-30 to the cytotoxic agent monomethyl auristatin $\mathrm{E}$ (MMAE) via a valinecitrulline peptide linker. Upon administration and internalization 
by CD30-positive tumor cells, brentuximab vedotin undergoes enzymatic cleavage, releasing MMAE into the cytosol; MMAE binds to tubulin and inhibits tubulin polymerization, which may result in G2/M phase arrest and tumor cell apoptosis. Transiently activated during lymphocyte activation, CD30 (tumor necrosis factor receptor superfamily, member 8 , (TNFRSF8)) may be constitutively expressed in hematologic malignancies including Hodgkin lymphoma and some T-cell NHLs. The linkage system in brentuximab vedotin is highly stable in plasma, resulting in cytotoxic specificity for CD30-positive cells.

The brentuximab vedotin is a CD30-directed antibody-drug conjugate indicated for the treatment of patients with systemic anaplastic large cell lymphoma after the failure of at least one prior multi-agent chemotherapy regimen. The most common adverse effects are neutropenia, peripheral sensory neuropathy, fatigue, nausea, anemia, upper respiratory tract infection, diarrhea, pyrexia, rash, thrombocytopenia, cough, and vomiting. Brentuximab vedotin can cause peripheral neuropathy.If patients experiences StevensJohnson syndrome than Brentuximab vedotin should be discontinued. Brentuximab vedotin can cause fetal harm, so, it should not be administered into pregnant women.

Non-FDA approved MABs: There are some MABs that are not currently approved by FDA for NHL and are under clinical trials in phase I-III as in the Table 1.

\section{Proteasome inhibitor immunotherapy}

Bortezomib [18]: A dipeptide boronic acid analogue with anti-neoplastic activity. Bortezomib reversibly inhibits the $26 \mathrm{~S}$ proteasome, a large protease complex that degrades ubiquinated proteins. By blocking the targeted proteolysis normally performed by the proteasome, bortezomib disrupts various cell signaling pathways, leading to cell cycle arrest, apoptosis and inhibition of angiogenesis. Specifically, the agent inhibits nuclear factor (NF)-kappa B, a protein that is constitutively activated in some cancers, thereby interfering with NF-kappa B-mediated cell survival, tumor growth and angiogenesis. In vivo, bortezomib delays tumor growth and enhances the cytotoxic effects of radiation and chemotherapy. Bortezomib is a proteasome inhibitor also indicated for the treatment of patients with multiple myeloma. The volume of distribution of Bortezomib is around 1884 $\mathrm{L} / \mathrm{m} 2$ and $83 \%$ bound to human plasma proteins. It is metabolized through CYP 2D6.

It has been approved by FDA for patients with mantle cell lymphoma who have been progressed after receiving at least one line of chemotherapy
The most common adverse effects are anorexia, peripheral neuropathy, vomiting, nausea, diarrhea, psychiatric disorders, constipation, asthenic conditions, neuralgia, neutropenia, decreased appetite, leukopenia,anemia, and pyrexia. Bortezomib should be discontinued in case of Reversible Posterior Leukoencephalopathy Syndrome (RPLS). Bortezomib can cause nausea, vomiting, diarrhea and constipation. The hypotension and various cardiac disorders occur throughout the therapy.

Non-FDA approved proteasome inhibitor: The proteasome inhibitor that is not currently approved by the FDA for NHL and under phase I is in Table 2.

\section{Kinase inhibitor immunotherapy}

Ibrutinib [20]: An orally bioavailable, small-molecule inhibitor of Bruton's tyrosine kinase (BTK) with potential anti-neoplastic activity. Upon oral administration, ibrutinib binds to and irreversibly inhibits BTK activity, thereby preventing both B-cell activation and B-cell-mediated signaling. This leads to an inhibition of the growth of malignant B cells that over express BTK. BTK, a member of the SRCrelated BTK/Tec family of cytoplasmic tyrosine kinases, is required for $\mathrm{B}$ cell receptor signaling, plays a key role in B-cell maturation and is over expressed in a number of B-cell malignancies. The expression of BTK in tumor cells is also associated with increased proliferation and survival. Imbruvica (Ibrutinib) is a kinase inhibitor, indicated for the treatment of patients with MCL who has received at least one prior therapy and for CLL who have received at least one prior therapy. The clearance rate of Ibrutinib is $1000 \mathrm{~L} / \mathrm{h}$ and half-life is $4-6$ hours.

The most common adverse effects are anorexia, peripheral neuropathy, vomiting, nausea, diarrhea, psychiatric disorders, constipation, asthenic conditions, neuralgia, neutropenia, decreased appetite, leukopenia and anemia, pyrexia, thrombocytopenia, musculoskeletal pain, and upper respiratory tract infection. The renal toxicities, anemia, thrombocytopenia and neutropenia take place in the treatment with Ibrutinib. Ibrutinib can cause fetal harm. It should be avoided in pregnant women.

Idelalisib [21]: An orally bioavailable, small molecule inhibitor of the delta isoform of the $110 \mathrm{kDa}$ catalytic subunit of class I phosphoinositide-3 kinase (PI3K) with potential immunomodulating and anti-neoplastic activities. Idelalisib inhibits the production of the second messenger phosphatidylinositol-3,4,5-trisphosphate (PIP3), preventing the activation of the PI3K signaling pathway and inhibiting tumor cell proliferation, motility and survival. Unlike other isoforms of PI3K, PI3K-delta is expressed primarily in hematopoietic lineages. The targeted inhibition of PI3K-delta is designed to preserve PI3K signaling

Table 1. Non-FDA approved MAB drugs [12-17].

\begin{tabular}{|l|l|l|l|}
\hline Drug & Clinical trial identifier number & Phase & Study Design \\
\hline Alemtuzumab & NCT00051701 & Phase 1,2 & $\begin{array}{l}\text { Non-Randomized, Open label, Safety/ } \\
\text { Efficacy Study }\end{array}$ \\
\hline Ipilimumab & NCT01769222 & Open label, Safety Study \\
\hline Yttrium Y 90 Ibritumomab Tiuxetan & NCT00577278 & Phase 1,2 & Open label, Safety/Efficacy Study \\
\hline Iodine I 131 monoclonal antibody BC8 & NCT00860171 & Phase 1,2 & Open label, Safety Study \\
\hline $\begin{array}{l}\text { Indium In 111 anti-CD45 monoclonal } \\
\text { antibody BC8 }\end{array}$ & NCT01678443 & Phase 1 & Open label, Safety Study \\
\hline Inotuzumab & NCT01925131 & Phase 1 & Open label, Safety Study \\
\hline
\end{tabular}

Table 2. Non-FDA approved proteasome inhibitor drugs [19].

\begin{tabular}{|l|l|l|l|}
\hline Drug & Clinical trial identifier number & Phase & Study Design \\
\hline Carfilzomib & NCT02187133 & Phase 1 & Open label, Safety Study \\
\hline
\end{tabular}


in normal, non-neoplastic cells.

The drug is indicated in relapsed follicular B-cell NHL (FL) in patients, who have received at least two prior systemic therapies. Relapsed small lymphocytic lymphoma (SLL) in patients, who have received at least two prior systemic therapies. It is metabolized through CYP3A and the clearance rate at steady state is $14.9 \mathrm{~L} / \mathrm{hr}$ and the half-life is 8.2 hours. The most common adverse effects are rash, nausea, diarrhea, fatigue, cough, abdominal pain, pyrexia, chills, and pneumonia. Idelalisib increases fatal and/or serious hepatotoxicity. Idelalisib causes diarrhea and colitis, so it should be avoided in NHL. Idelalisib causes fatal and serious pneumonitis. Idelalisib can cause fetal harm. It should be avoided in pregnant women.

Non-FDA approved kinase inhibitors: Few kinase inhibitors that are under clinical trials in phase I, II and III are in the Table 3.

\section{Histone deacetylases (HDACs) inhibitors immunotherapy}

Vorinostat [27]: A synthetic hydroxamic acid derivative with anti-neoplastic activity. Vorinostat, a second generation polarplanar compound, binds to the catalytic domain of the HDACs. This allows the hydroxamic moiety to chelate zinc ion located in the catalytic pockets of HDAC, thereby inhibiting deacetylation and leading to an accumulation of both hyperacetylated histones and transcription factors. Hyperacetylation of histone proteins results in the upregulation of the cyclin-dependant kinase p21, followed by G1 arrest. Hyperacetylation of non-histone proteins such as tumor suppressor p53, alpha tubulin and heat-shock protein 90 produces additional anti-proliferative effects. This agent also induces apoptosis and sensitizes tumor cells to cell death processes. Vorinostat crosses the blood-brain barrier. The half-life is approximately two hours.

Vorinostat is a HDAC inhibitor indicated for the treatment of cutaneous manifestations in patients with cutaneous $\mathrm{T}$-cell lymphoma (CTCL), who have progressive, persistent or recurrent disease on or following two systemic therapies. The most common adverse effects are taste disorders such as dry mouth and dysgeusia, constitutional symptoms such as chills and fatigue, gastrointestinal symptoms such as nausea, vomiting, diarrhea, constipation, weight loss and anorexia, and hematologic abnormalities, such as anemia and thrombocytopenia. Vorinostat can cause fetal harm. It should be avoided in pregnant women.

Belinostat [28]: A novel hydroxamic acid-type HDAC inhibitor with anti-neoplastic activity. Belinostat targets HDAC enzymes, thereby inhibiting tumor cell proliferation, inducing apoptosis, promoting cellular differentiation and inhibiting angiogenesis. This agent may sensitize drug-resistant tumor cells to other anti-neoplastic agents, possibly through a mechanism involving the down-regulation of thymidylate synthase.

Belinostat is a HDAC inhibitor indicated for the treatment of patients with relapsed or refractory peripheral T-cell lymphoma (PTCL). This indication is approved under accelerated approval based on tumor response rate and duration of response. An improvement in survival or disease-related symptoms has not been established. Continued approval for this indication may be contingent upon verification and description of clinical benefit in the confirmatory trial.

The most common adverse effects are nausea, vomiting, anemia, pyrexia and fatigue. Anemia, leukopenia (lymphopenia and neutropenia) and thrombocytopenia occurs during the treatment with Belinostat. The fatal infections as well as sepsis and pneumonia occurs during the treatment with Belinostat so, it should not be administered with active infection. It can cause liver function abnormalities and fatal hepatotoxicity so, it should be permanently discontinued. Belinostat can cause fetal harm. It should be avoided in pregnant women.

\section{Cytokine therapy}

Denileukin diftitox [29]: A cytotoxic recombinant protein consisting of interleukin-2 (IL-2) protein sequences fused to diphtheria toxin. The IL-2 protein sequence moiety of denileukin difitox directs the cytocidal action of diphtheria toxin to cells that express IL-2 receptors. After the toxin moiety is internalized into target IL-2 receptor-expressing cells, its catalytic domain catalyzes the transfer of the ADP-ribose moiety of NAD to a post translationally modified histidine residue of elongation factor 2 (EF-2), called diphthamine. This covalent modification inactivates EF-2 and disrupts polypeptide chain elongation, resulting in cell death.

Denileukin Difitox is a CD25-directed cytotoxin, indicated for the treatment of patients with persistent or recurrent cutaneous T-cell lymphoma whose malignant cells express the CD25 component of the IL-2 receptor. The most common adverse effects are nausea, pruritus, dyspnea, diarrhea, fatigue, vomiting, cough, headache, pyrexia, peripheral edema and rigors. If the patients have Capillary leak syndrome, weight gain, hypotension and new onset or worsening edema, then the dose of Denileukin Difitox should be changed. The loss of visual acuity and typically with loss of color vision may occur during the treatment with Denileukin Difitox.

Interferon alfa-2b [30]: A non-glycosylated recombinant interferon with antiviral and anti-neoplastic activities. Alfa interferons bind to specific cell-surface receptors, resulting in the transcription and translation of genes, whose protein products mediate antiviral, antiproliferative, anticancer and immune-modulating effects.

Interferon alfa- $2 \mathrm{~b}$, recombinant for injection is indicated for the treatment of patients, 18 years of age or older with hairy cell leukemia. It is also indicated for the initial treatment of clinically aggressive follicular NHL in conjunction with anthracycline containing combination chemotherapy in patients of 18 years of age or older. The half-life is two hours.

Table 3. Non-FDA approved kinase inhibitor drugs [22-26].

\begin{tabular}{|c|c|c|c|c|}
\hline Drug & Clinical trial identifier number & Phase & Study Design & Number \\
\hline Imatinib mesylate & NCT00684411 & Phase 2 & Open label, Safety/Efficacy Study & $\begin{array}{l}\text { TKI KIT, Bcr-Abl, } \\
\text { PDGFR }\end{array}$ \\
\hline Sunitinib malate & NCT00392496 & Phase 2 & $\begin{array}{l}\text { Non-Randomized, Open label, Safety/Efficacy } \\
\text { Study }\end{array}$ & $\begin{array}{l}\text { VEGFR2, PDGFRb, } \\
\text { FLT3, c-kit }\end{array}$ \\
\hline Dasatinib & NCT00550615 & Phase 1,2 & Non-Randomized, Open label, Safety/Efficacy Study & Bcr-Abl kinase \\
\hline Sorafenib & NCT00278382 & Phase 2 & Open label, Efficacy Study & $\begin{array}{l}\text { VEGFR-2/PDGFR- } \\
\text { beta signaling }\end{array}$ \\
\hline ACP-196 & NCT02337829 & Phase 2 & Randomized, Safety/Efficacy Study, Open Label & BTK \\
\hline
\end{tabular}


The most common adverse effects are vomiting, nausea, anorexia, fever, fatigue and headache. Hepatotoxicity as well as fatality may occur during the treatment with Interferon alfa-2b, so it should be discontinued. If patients experienced pneumonia, pneumonitis, pulmonary infiltrates as well as fatality throughout the treatment, then it should be discontinued. Throughout the treatment, it the patient can develop different thyroid abnormalities either hyperthyroid or hypothyroid.

\section{Immunomodulators}

Lenalidomide [31]: A thalidomide analog with potential antineoplastic activity. Lenalidomide inhibits TNF-alpha production, stimulates $\mathrm{T}$ cells, reduces serum levels of the cytokines vascular endothelial growth factor (VEGF) and basic fibroblast growth factor (bFGF) and inhibits angiogenesis. This agent also promotes G1 cell cycle arrest and apoptosis of malignant cells. The AUC of Lenalidomide is $57 \%$ and its plasma protein binding is $30 \%$. The half-life is around 3 hours.

Lenalidomide is approved for the treatment of patients with mantle cell lymphoma, whose disease has relapsed or progressed after two prior therapies, one of which included bortezomib.

The most common adverse effects are myelodysplastic syndromes, cardiac disorders, gastrointestinal disorders, ear and labyrinth disorders, injury, poisoning and procedural complications, immune system disorders, endocrine disorders, infections and infestations, hepatobiliary disorders, and immune system disorders such as hypersensitivity. Lenalidomide can cause fetal harm. It should be avoided in pregnant women. The patients experience hematologic toxicity such as thrombocytopenia and neutropenia during the treatment, so the dose of Lenalidomide should be changed.

Non-FDA approved immunomodulators: There are some immunomodulators that are not currently approved by the FDA for NHL. These are under clinical trials in phase I, II and III as in the Table 4.

\section{Mammalian target of rapamycin (mTOR) immunotherapy}

The mammalian target of rapamycin (mTOR) is an intracellular serine/threonine kinase that exists as a downstream component of numerous signaling pathways. The activation of mTOR results in the production of proteins involved in cell metabolism, growth, proliferation, and angiogenesis. Aberrant activation of mTOR signaling has been identified in a number of cancers, and targeted inhibition of mTOR has been successful in achieving tumor responses, prolonging progression-free survival, and increasing overall survival in various oncologic patient populations.

There is no mTOR inhibitor that is not currently approved by FDA for NHL. The mTOR inhibitors are under clinical trials in phase I, II and III are in the Table 5.

\section{Stem cell transplantations [2]}

Generally, there are two basic types of transplantations such as Autologous (the cells come from the patient itself) and Allogeneic (the cells come from a matched related or unrelated donor). Both these methods are used for the treatment of Hodgkin lymphoma.

1. Autologous Stem Cell Transplantations: It utilizes the own stem cells of the patient, isolated from the bone marrow or the blood and is put to freeze. After the intensive chemotherapy and/or radiation therapy, these cells are re-infused into the patient.

2. Allogenic Stem Cell Transplantations: In this type of transplantation, the stem cells are obtained from another person whose HLA type closely resembles to that of the patient. The most successful donors are often a close relative, or more specifically a brother or a sister. If the HLA of close relatives does not match, stem cells can be obtained from a matched unrelated donor (MUD). However, use of such stem cells might lead to several complications. Umbilical cord stem cells can also be used.

\section{Miscellaneous drugs}

Few other therapies that are not approved by FDA are listed below in the Table 6.

\section{Conclusion}

Immunotherapy has proven to be effective in the treatment of NHL. Our success in treating NHL is increasing and advancing with the knowledge of the function of the immune system. Researchers are still challenged in exploring innate and adaptive immune systems. Immunotherapy has been a promising development in the past few years. The recent activities have increased our understanding of the tumor microenvironment, various immunotherapeutic modalities or combination therapy (like chemotherapy with immunotherapy). Additionally, the effects of such modalities in combination with immunotherapy in cancer patients are still exploratory phase. The

Table 4. Non-FDA approved immunomodulators [32].

\begin{tabular}{|l|l|l|l|}
\hline Drug & Clinical trial identifier number & Phase & Study Design \\
\hline Thalidomide & NCT00022581 & Phase 2 & Open label, Efficacy Study \\
\hline
\end{tabular}

Table 5. Non-FDA approved mTOR drugs [33,34].

\begin{tabular}{|l|l|l|l|}
\hline Drug & Clinical trial identifier number & Phase & Study Design \\
\hline Temsirolimus & NCT01076543 & Phase 1,2 & Open label, Safety/Efficacy Study \\
\hline Everolimus & NCT00918333 & Phase 1,2 & Open label, Safety/Efficacy Study \\
\hline
\end{tabular}

Table 6. Non-FDA approved miscellaneous drugs [35-37].

\begin{tabular}{|l|l|l|l|}
\hline Drug & Clinical trial identifier number & Phase & Study Design \\
\hline Interleukin 2 & NCT00968760 & Phase 1 & $\begin{array}{l}\text { Non-Randomized, Safety/Efficacy Study, Open } \\
\text { Label }\end{array}$ \\
\hline Alisertib & NCT01567709 & Phase 1 & Safety/Efficacy Study, Open Label \\
\hline $\begin{array}{l}\text { Autologous CD19CAR-CD28-CD3zeta- } \\
\text { EGFRt-expressing Tcm-enriched T cells }\end{array}$ & NCT02153580 & Phase 1 & Safety Study, Open Label \\
\hline
\end{tabular}


complete perspective of immunotherapy treatment has not been realized or utilized. Proper preclinical and clinical designs are the important pillars in understanding the future of immunotherapy in treating cancer patients.

\section{References}

1. SEER Stat Fact Sheets: Non-Hodgkin Lymphoma (2013) Surveillance, Epidemiology, and End Results (SEER). Cancer Statistics.

2. Non-Hodgkin Lymphoma. American Cancer Society.

3. Kath R, Donhuijsen K, Hayungs J, Albrecht K, Seeber S, et al. (1995) Primary gastric non-Hodgkin's lymphoma: a clinic pathological study of 41 patients. Journal of Cancer Research and Clinical Oncology 121: 51-56.

4. Ekstrom-Smedby K (2006) Epidemiology and etiology of non-Hodgkin lymphoma-a review. Acta Oncol 45: 258-271. [Crossref]

5. Downing JR, Shurtleff SA, Zielenska M, Curcio-Brint AM, Behm FG, et al. (1995) Molecular detection of the $(2 ; 5)$ translocation of non-Hodgkin's lymphoma by reverse transcriptase polymerase chain reaction. Blood 85: 3416-3422. [Crossref]

6. Rituximab Infusion (2012) Drugs, FDA.

7. FDA (2014) Approved label ARZERRA (ofatumumab) Manufactured by glaxo group limited.

8. FDA (2013) Approved label GAZYVA (obinutuzumab) Manufactured by Genentech, Inc.

9. FDA (2001) Approved labelZEVALINTM (Ibritumomab Tiuxetan) Manufactured by IDEC Pharmaceuticals Corporation.

10. FDA (2003) approved label BEXXAR $®$ (Tositumomab and Iodine I 131 Tositumomab) Manufactured by Corixa Corporation.

11. FDA (2011) Approved label ADCETRISTM (brentuximab vedotin). Manufactured by Seattle Genetics, Inc.

12. Genzyme (2015) Phase I/II Study of CAMPATH in Patients With Relapsing or Refractory Non-Hodgkin's Lymphoma. In: ClinicalTrials.gov Bethesda (MD), National Library of Medicine (US).

13. Non-Hodgkin Lymphoma (2015) Ipilimumab and Local Radiation Therapy in Treating Patients with Recurrent Melanoma, Colon, or Rectal Cancer. In: ClinicalTrials. gov, Bethesda (MD): National Library of Medicine Stanford University; Stanford University, US.

14. Shimoni A, Nagler A (2007) Radioimmunotherapy and stem-cell transplantation in the treatment of aggressive B-cell lymphoma. Leukemia \& lymphoma 48: 2110-2120.

15. Fred Hutchinson Cancer Research Center (2015) Iodine I 131 Monoclonal Antibody BC8 Before Autologous Stem Cell Transplant in Treating Patients With Relapsed or Refractory Hodgkin Lymphoma or Non-Hodgkin Lymphoma. In: ClinicalTrials.gov.

16. Fred Hutchinson Cancer Research Center (2015) Monoclonal Antibody Therapy Before Stem Cell Transplant in Treating Patients With Relapsed or Refractory Lymphoid Malignancies. In: ClinicalTrials.gov.

17. Southwest Oncology Group (2015) S1312, Inotuzumab Ozogamicin and Combination Chemotherapy in Treating Patients with Relapsed or Refractory Acute Leukemia. In: ClinicalTrials.gov.
18. FDA (2008) Approved label VELCADE (bortezomib) Manufactured byMillennium Pharmaceuticals.

19. University of California (2015) C Babis Andreadis, University of California, San Francisco. In: ClinicalTrials.gov.

20. FDA (2014) Approved label IMBRUVICATM (ibrutinib). Manufactured by Pharmacyclics.

21. FDA (2014) Approved label ZYDELIG (idelalisib) Manufactured by Gilead Sciences.

22. Dana-Farber Cancer Institute (2015) Gleevec in Relapsed/Refractory T cell NonHodgkin's Lymphoma. Eric Jacobsen, MD, Dana-Farber Cancer Institute. In: ClinicalTrials.gov.

23. National Cancer Institute (NCI) (2015) Sunitinib in Treating Patients with Relapsed or Refractory Diffuse or Mediastinal Large B-Cell Lymphoma. In: ClinicalTrials.gov.

24. University of Nebraska (2015) Dasatinib in Relapsed or Refractory Non-Hodgkin's Lymphoma. In: ClinicalTrials.gov.

25. National Cancer Institute (NCI) (2015) Sorafenib in Treating Patients with Recurrent Non-Hodgkin's Lymphoma. In: ClinicalTrials.gov.

26. Acerta Pharma BV (2015) ACP-196 in Patients With Relapsed/Refractory and Treatment naïve Deletion 17p CLL/SLL. In: ClinicalTrials.gov.

27. FDA (2011) Approved label ZOLINZA ${ }^{\circledR}$ (Vorinostat) Capsules Manufactured by Patheon.

28. FDA (2014) Approved label BELEODAQ ${ }^{\circledR}$ (Belinostat) Manufactured by Spectrum Pharmaceuticals.

29. FDA (2008) Approved label ONTAK ${ }^{\circledR}$ (denileukin diftitox) Manufactured by Eisai Medical Research

30. FDA (1997) Approved label INTRONB A (interferon alfa-2b) Manufactured by Schering Corporation.

31. FDA (2008) Approved label REVLIMID ${ }^{\circledR}$ (lenalidomide) Manufactured by Celgene Corporation.

32. National Cancer Institute (NCI) (2015) Thalidomide in Treating Patients with Relapsed or Refractory Non-Hodgkin's Lymphoma. In: ClinicalTrials.gov.

33. National Cancer Institute (NCI) (2015) Lenalidomide and Temsirolimus in Treating Patients with Relapsed or Refractory Hodgkin Lymphoma or Non-Hodgkin.

34. Mayo Clinic (2015) Panobinostat and Everolimus in Treating Patients With Recurrent Multiple Myeloma, Non-Hodgkin Lymphoma, or Hodgkin Lymphoma. In: ClinicalTrials.gov.

35. Anderson MD (2015) Autologous CD19-specific T Cells Infusion. In: ClinicalTrials gov.

36. National Cancer Institute (NCI) (2015) Alisertib in Combination With Vorinosta in Treating Patients With Relapsed or Recurrent Hodgkin Lymphoma, B-Cell NonHodgkin Lymphoma, or Peripheral T-Cell Lympho. In: ClinicalTrials.gov.

37. City of Hope Medical Center (2015) Cellular Immunotherapy Following Cyclophosphamide in Treating Patients with Recurrent Non-Hodgkin Lymphomas, Chronic Lymphocytic Leukemia or B-Cell Prolymphocytic Leukemia. In: ClinicalTrials.gov.

Copyright: (C2016 Khoni NS. This is an open-access article distributed under the terms of the Creative Commons Attribution License, which permits unrestricted use, distribution, and reproduction in any medium, provided the original author and source are credited. 